Revue d'histoire de l'Amérique française

TALEVUE D.HISTOIRE DE L'AMÉRIQUE FRANÇAISE

\title{
Correspondance autour de la question scolaire du Nouveau-Brunswick 1873 (suite)
}

\section{Lionel Groulx}

Volume 4, numéro 3, décembre 1950

URI : https://id.erudit.org/iderudit/801656ar

DOI : https://doi.org/10.7202/801656ar

Aller au sommaire du numéro

Éditeur(s)

Institut d'histoire de l'Amérique française

ISSN

0035-2357 (imprimé)

1492-1383 (numérique)

Découvrir la revue

Citer ce document

Groulx, L. (1950). Correspondance autour de la question scolaire du

Nouveau-Brunswick 1873 (suite). Revue d'histoire de l'Amérique française, 4(3),

427-431. https://doi.org/10.7202/801656ar d'utilisation que vous pouvez consulter en ligne.

https://apropos.erudit.org/fr/usagers/politique-dutilisation/ 


\title{
DOCUMENTS INÉDITS
}

\section{CORRESPONDANCE AUTOUR DE LA QUESTION SCOLAIRE DU NOUVEAU-BRUNSWICK 1873}

\author{
(suite)
}

Nous continuons la publication des lettres de Cléophas Beausoleil à Alphonse Desjardins. Depuis la publication de la première série, deux autres lettres de Beausoleil sont tombées entre nos mains, l'une du 17 mars 187s, l'autre du 25 mars 1873. On est prié de leur restituer leur place dans cette correspondance. Nous nous excusons, en même temps, auprès de nos lecteurs d'une erreur qui s'est glissée dans notre courte biographie d'Alphonse Desjardins. Nous avions emprunté nos renseignements à un dictionnaire biographique. Le Père Paul Desjardins, s.j., veut bien nous fournir, sur un point, une rectification: Alphonse Desjardins n'est pas le fondateur du Nouveau-Monde. Il n'entra au bureau de direction, avec le titre de vice-président, qu'au mois de mai 1872, pour demeurer attaché au journal jusqu'au mois de juillet 1879, alors que Frédéric Houde lui succéda. Le véritable fondateur du Nouveau-Monde serait, croit le Père Desjardins, Messire Godefroy Lamarche qui s'adjoignit comme premier rédacteur Joseph Royal. Autre rectification: nous avions fait suivre d'un point d'interrogation le nom d'un M. Genoud qui figure dans la correspondance de Beausoleil. Le Père Desjardins nous apprend encore qu'il s'agit d'un M. Auguste Genand, avocat et journaliste, ancien étudiant à l'École de Droit du Collège Sainte-Marie à Montréal et qui a collaboré au journal l'Ordre.

Que le Père Desjardins veuille bien agréer ici nos remerciements.

L.G.

Mon cher Monsieur,

Ottawa, 17 mars 1873

Tout le monde s'occupe ici de la guerre qui s'est enfin déclarée entre Cauchon et Langevin, entre le Journal de Québec et toute la presse conservatrice. Le Journal doit contenir aujourd'hui en réponse à la Minerve un article de trois colonnes et demie. Cauchon a mis une après-midi et une nuit à l'écrire. Ce doit être quelque chose de féroce. Vous avez sans doute remarqué que le Courrier d'Outaouais regrette de ne nous avoir pas donné raison contre l'homme du Journal. C'est signe que la leçon commence à profiter. 
Cauchon déclare à qui veut l'entendre qu'il a commis une bévue lorsqu'il a attaqué le programme et les évêques qui l'appuyaient; que jamais il ne retombera dans la même faute et que c'est à la demande du gouvernement fédéral et du gouvernement local qu'il en a agi ainsi et qu'après les avoir servis et s'être cré des ennemis, il est abandonné et repoussé, méprisé et ridiculisé par ceux-mêmes pour lesquels il s'est sacrifié.

Nos amis, Armand, Bellerose et Masson sont unanimes à déclarer que le Nouveau Monde doit laisser ses ennemis s'entredéchirer. Lorsque la querelle tirera à sa fin, ce sera pour lui le temps d'intervenir et de demander aux deux partis ce qu'ils ont gagné à leur guerre odieuse contre les vrais principes conservateurs. S'il intervenait aujourd'hui, il courrait le risque de voir se renouveler la fable de l'homme qui battait sa femme et qu'un voisin voulut arrêter dans sa brutalité. L'homme et la femme lui tombèrent dessus et le chassèrent à grands coups. Je partage entièrement cette opinion. Qu'en pensez-vous?

Un autre sujet de conversation, c'est le discours de Chapleau à l'élection de Terrebonne et l'ovation qui lui a été faite au Séminaire de StoThérèse. Que dites-vous de jeunes gens qui promettent de suivre plus tard les grands exemples de Chapleau? Ne lui tomberez-vous point dessus pour son discours et l'affichement qu'il a fait de son mépris du programme et des principes qu'il consacre?

L'attitude politique que vous définissez dans votre dernière lettre me va parfa tement et je concours pleinement dans le programme que vous y tracez. Elle a toujours été dans ma pensée la seule ligne de conduite raisonnable qu'un journal comme le $N$. Monde devait suivre.

Avez-vous l'intention de publier la circulaire de Mgr de Montréal dont vous me parlez dans votre lettre? Sinon, faites-moi done le plaisir de m'en expédier un exemplaire.

Il est rumeur ici que le Journal des Trois-Rivières a reçu un bref du St Père. C'est Magloire McLeod qui l'a écrit à son frère qui est ici employé au recensement.

La semaine dernière - avant que la Congrégation ait siégé - la nouvelle s'est répandue ici, venant de Langevin, car Langelier - le grand niais de six pieds quatre pouces - disait le tenir d'une haute autorité, que la Propagande avait rendu sa décision finale dans les affaires du Canada; que la question de l'Université ainsi que celle des Paroisses étaient renvoyées à un concile provincial; que le $N$. Monde avait reçu une très mauvaise note et que la Comédie infernale était réfórée à l'évêque d'Outaouais. Cette rumeur avait cré une immense sensation et jetté le découragement chez nos amis. La nouvelle du Canadien que la Congrégation n'avait commencé à siéger que le 13 prouve que la rumeur était fausse et remet chaque chose à sa place.

Auriez-vous la complaisance d'envoyer le Nouveau-Monde à Madame Beausoleil, au No 76 Rue St André, d'ici à mon retour? Vous me feriez, et à elle aussi, un grand plaisir. 
Notre ami Genand est en bonne santé et vous salue. Voici la mystification dont il a été victime chez le Dr St Jean, d'Ottawa.

Il se trouvait l'autre jour en compagnie et Madamo St Jean lui demanda s'il irait faire visite à Madame Hamel. Genand ne comprit pas du coup et le Dr Paquet lui répéta la question, mais avec abréviation comme suit Allez vous voir Mam $\mathrm{Hamel}$ ? (ma mamelle). Genand ne comprit pas davantage et le Dr lui dit assez haut: Allez-vous voir Madame Hamel?

Notre ami s'ecria: - J'allais donc le dire pour qu'on le susse?

Imaginez l'éclat de rire homérique qui accueillit cette repartie que Genand avait faite bien innocemment.

Présentez mes amitiés à M. le Chanoine Lamarche et à M. Laferrière.

Tout à vous

C. Beausoleil

Mon cher Monsieur,

Ottawa, 25 mars 1873

Le Courrier d'Outaouais annonce ce matin qu'il a reşu de Montréal une dépêche annonçant que l'Archevêque a gagné toutes ses causes à Rome. D'un autro côté Duvernay a montré à Masson une dépêche de Québec dans laquelle on annonçait que l'Archevêque avait télégraphié que Mgt de Montréal avait été condamné ainsi que le Nouveau Monde. Nos amis sont très anxieux de savoir si vous avez d'autres nouvelles de Rome par télégraphe ou par la malle. Je leur ai dit ce que vous m'en aviez appris; mais ils sont très anxieux de tout savoir. C'est à leur demande que je vous écris ce qui précède.

Le ministère a éprouvé hier $s$ jir une petite défaite qui lui est fort sensible. Bellerose m'a dit qu'il regrettait de n'avoir point voté avec l'opposition pour mieux prouver que ce n'était point un vote de parti. Il ne s'attendait pas du tout à ce résultat.

Demain c'est l'affaire de l'officier-rapporteur du district électoral de Muskoka qui occupera la Chambre; j'aurai le bill pour abolir le double mandat. Tout le monde s'attend qu'il sera adopté, mais on estime diversement la majorité de 10 à 25 voix. Je ne vois pas du tout ce qu'il en sera. Vendredi jour du gouvernement, la Chambre discutera des bills de $M$. Mitchel.

Dans quelques jours, si les négociations en vue d'obtenir l'amnistie pour Riel viennent à échouer, je vous transmettrai toute la correspondance à ce sujet dont Masson m'a promis la primeur.

Cette question promet d'être fort sérieu $3 e$, si elle n'est pas décidée dans le sens que réclament la justice, la parole donnée et l'honneur.

Bellerose est tout disposé à marcher dans la question des Écoles et dans celle de.s Better terms. Pourvu que cela ne change pas d'ici à quelques jours!... 
Je vous aurais dejà entretenu du Pacifique si les papiers se trouvaient devant la Chambre. Mais ainsi que je l'ai dit dans our correspondence, tout est entre les mains de l'Imprimeur do la Reine qui se trouvant en banqueroute ou sur le point de le devenir, ne se presse pas d'exécuter les impressions de la Chambre, espérant les forcer à lui accorder une indemnité.

D'un autre côté, écrire sans connaître les choses, c'est s'exposer à bien des mécomptes.

Comment vont nos amis Rivard et Drolet? Vont-ils enfin se décider à faire quelque chose? Je vois que celui-ci vient de faire l'acquisition de deux nouvelles propriétés. J'ai pensé que c'était mauvais signe.

Je vous félicite de vos articles sur Chapleau et les autres. Ils ont été loués par tous nos amis.

$J$ 'espère que vous ne doutez point que je suis en faveur du Pacifique et de la compagnie Allan, à moins que les documents révèlent des choses tout à fait extraordinaires qu'il n'est guère possible de prévoir. J'ai assez 6crit dans le passé sur ce sujet pour que mon opinion soit bien connue.

Rien de nouveau ici. Nos amis vous saluent cordialement ainsi que Genand, Dupras, etc.

Mes amitiés à M. Lamarche, à M. Laferrière, à Mr et Madame Plinguet.

Tout à vous,

C. Beausoleil

Vous pouvez m'adresser vos lettres directement. Elles viennent franches de port à toute adresse à la chambre des Communes.

C.B.

Mon cher Monsieur,

Ottawa, 3 avril 1873

J'accuse réception de la somme de $\$ 30.00$ que vous avez bien voulu m'expédier et je vous prie d'agréer mes remerciements. Si je ne me trompe, j'ai reçu de vous a/c les sommes suivantes. Quatre semaines se sont écoulées, depuis mon départ à venir à samedi dernier.

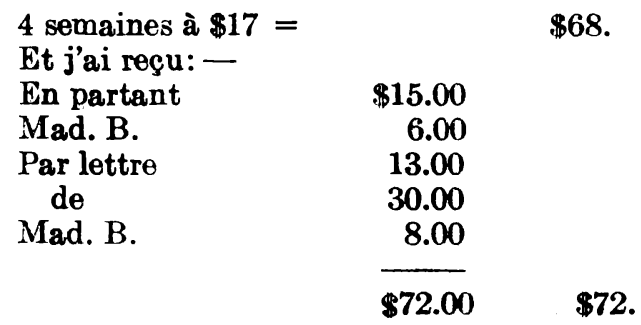

Laissant en votre faveur à la date de samedi dernier une balance de $\$ 4.00$. 
Lorsque j'ai reçu votre lettre, vous saviez le résultat de la motionHuntingdon. Jamais je n'ai vu ni entendu parler d'une tactique si stupide ou plutót d'un manque de tactique aussi absolu. C'est quelque-chose d'inoui que la proposition d'une motion de non-confiance sans l'accompagner d'un seul mot de commentaires. Je doute fort maintenant que le gouvernement court des dangers sérieux et je suis persuadé que Sir John trouvera bien le moyen de tourner les difficultés qui pourront se présenter à l'avenir sur son chemin. C'est un écrasement terrible et l'opposition est restée atterrée du résultat. Il était pourtant facile de le prévoir. Cette après-midi sir John a donné avis qu'il proposerait la formation d'un comité chargé de s'enquérir des accusations portées contre le gouvernement. L'Opposition voulait ramener l'affaire sous une autre forme; mais elle est tournée, démoralisée, battue, elle a perdu confiance et elle peut dire adieu à ses beaux rêves, à moins que le ministère commette quelque grosse maladresse, ce qui n'est pas probable.

M. le curé Labelle est ici et il continue son double jeu, parlant tantôt pour l'évêque et tantôt pour le séminaire, suivant celui auquel il s'adresse. Il me paraît très impopulaire parmi les membres.

J'espère pouvoir vous envoyer demain un article sur la politique fiscale du pays. Il paraît qu'ayant exposé à $M$. Masson et à quelques autres mes opinions sur ce sujet pour voir comment elles seraient vues, et m'assurer qu'elles étaient exactes, deux députés ministériels - parmi lesquels $M$. Gendron - sont allés dire à Langevin que je parlais contre le budget, mêlant l'ignorance la plus crasse à l'honorable métier d'espion et de délateur. Cela m'irrite d'abord intérieurement, mais je finis par en rire de bon cœur. J'ai l'intention de faire des articles sérieux appuyés d'autorités que ces savants MM ne pourront même pas récuser.

$J_{\theta}$ vous remercie des renseignements que vous me donnez sur Robillard; je vais prendre la chose en considération, comme dirait M. Chauveau. A propos, que dites-vous des révélations de Fabre sur le compte de Chauveau et de Montpetit. A table j'ai rappelé à Ls. Archambault que c'était son nouveau collègue Chapleau qui devait amener l'affaire devant la Chambre, si Fabre avait voulu publier les lettres de Montpetit. Tous gens bien respectables qui s'estiment à leur valeur!

Nous attendons toujours les nouvelles de Rome avec anxiété et nous nous réjouissons beaucoup qu'elles soient f'avorables.

Veuillez présenter mes hommages à M. le Chanoine Lamarche, mes amitiés à $M$. Laferrière et me croire.

Votre ami tout dévoué,

C. Beausoleil

P.S. J'ai eu cette après-midi une longue conversation avec Royal à propos des affaires de Manitoba. Il paraît que tout y va très mal.

(à suivre) 